\title{
REVIEW
}

\section{Angiogenesis, thrombospondin, and ductal carcinoma in situ of the breast}

\section{A Rice, C M Quinn}

J Clin Pathol 2002;55:569-574

Angiogenesis, the growth of new vessels from existing vasculature, plays an essential role in tumour development. The process involves interaction between a variety of cells, growth factors, and components of the extracellular matrix, regulated by pro-angiogenic and anti-angiogenic factors. This review profiles these factors, outlines the available methods for measuring new vessel formation, and discusses the importance of angiogenesis in breast cancer, with emphasis on ductal carcinoma in situ.

See end of article for authors' affiliations

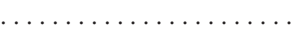

Correspondence to: Dr A Rice, Royal Brompton Hospital, Sydney Street, London SW3, UK. ricealex@hotmail.com

Accepted for publication 19 February 2002
A ngiogenesis is the growth of new vessels from existing vasculature that occurs during development and in vascular remodelling in the adult. It plays an essential role in tumour progression, providing nutrients and growth factors, and a means of tumour cell dissemination. Angiogenesis is a multistep process, which involves changes in the extracellular matrix, and endothelial cell proliferation, migration, and differentiation into capillaries. Studies of tumour biology reveal a complex network of autocrine and paracrine interactions between tumour cells, stromal cells, and endothelial cells, which are in turn influenced by the composition of the extracellular matrix. It has become increasingly apparent over the past few years that new vessel growth depends on the net effect of a variety of pro-angiogenic and anti-angiogenic molecules, including growth factors and components of the extracellular matrix. Not only does the extracellular matrix act as a scaffold for capillary growth, but it can bind and influence the availability of angiogenic molecules and influence the cell cycle through the binding of cell surface receptors. The study of angiogenesis and its role in tumour biology has produced a large body of work in the literature and has resulted in trials of new anti-angiogenic drugs in the treatment of cancer. $^{12}$

\section{ANGIOGENESIS}

The microvasculature is a dynamic system that plays an important role in a variety of physiological and pathological processes and it switches between quiescent and activated states. New vessels can arise by several processes: angiogenesis, vascular remodelling, and the recruitment of endothelial precursor cells from bone marrow and blood vessels. ${ }^{3}$ Angiogenesis is a multistep process that depends upon cooperation and interaction between a variety of cells, growth factors, and components of the extracellular matrix. It requires the destabilisation of existing vessels, increased permeability with extravasation of plasma proteins and enzymes into the surrounding stroma, changes in endothelial cell adhesion with endothelial cell migration, proliferation, survival, and stabilisation of newly formed vascular channels. Vascular remodelling describes new vessel formation by the insertion of interstitial tissue columns into the vessel lumen, with subsequent growth of these columns and partitioning of the vessel. This process of intussusception occurs in normal developing organs and has been shown to occur in colonic adenocarcinoma. ${ }^{4}$ Co-option of endothelial progenitor cells from the circulation into new vessels is known to occur in development, but may also occur in adults. ${ }^{5}$

Angiogenesis is recognised as a key factor in the progression of invasive tumours, as enunciated in the "angiogenesis progression" hypothesis. ${ }^{6}$ The pathways controlling the switch to an angiogenic phenotype in tumours are complex and poorly characterised but include hypoxia, genetic mutation, and stromal and inflammatory cell responses. There is evidence that changes in oncogene and tumour suppressor gene expression influence new vessel growth during tumour progression. ${ }^{7}$ For example, mutations in the tumour suppressor genes p53 and VHL result in the loss of expression of the anti-angiogenic factor thrombospondin 1 (TSP-1) ${ }^{8}$ and increased expression of the angiogenic factor vascular endothelial growth factor (VEGF), ${ }^{9}$ respectively.

In many tumours, including breast cancer, areas of increased tumour cell proliferation are associated with areas of increased microvessel density ("hot spots"). ${ }^{10}$ Not only do new vessels supply oxygen and nutrients to metabolically active tumour cells, but there is strong evidence for the presence of reciprocal interactions between tumour cells and endothelial cells. In addition to angiogenic factors such as VEGF, activated endothelial cells can produce factors that influence tumour cell growth and invasion-for example, hepatocyte growth factor, basic fibroblast growth factor (bFGF), and extracellular proteases. ${ }^{611}$

Inflammatory and stromal cells are also a source of angiogenic factors and extracellular

Abbreviations: bFGF, basic fibroblast growth factor; CIN, cervical intraepithelial neoplasia; DCIS, ductal carcinoma in situ; MMP, matrix metalloprotease; TF, tissue factor; TSP, thrombospondin; VEGF, vascular endothelial growth factor; VEGFR, vascular endothelial growth factor receptor 
proteases. For example, macrophages can synthesise VEGF, tumour necrosis factor $\alpha$, bFGF, interleukin 8 and the anti-angiogenic factor TSP-1. In addition, they produce extracellular proteases and elements of the coagulation system, such as tissue factor (TF) and thrombin, which play a role in vessel growth. ${ }^{12} 13$

Two studies investigated the expression of multiple growth factors and their receptors in the tumour, endothelial, and stromal compartments of breast cancer, and have identified potential autocrine and paracrine loops in vivo. In addition, correlations between the presence of these loops and tumour proliferation were found..$^{14}{ }^{15}$ These data highlight the importance of studying multiple factors within the tumour milieu and suggest a possible role for computer modelling of these complex processes.

\section{ANGIOGENIC AND ANTI-ANGIOGENIC FACTORS}

Growth factors specific to angiogenesis include VEGF and the angiopoietins. VEGF expression is induced by hypoxia and a variety of oncogenes, including ras and erbB, and is inhibited by the tumour suppressor gene VHL. VHL is structurally related to other growth factors including VEGF-C (a subtype of VEGF) and placental growth factor. There are three VEGF receptors, two of which are present on endothelial cells (VEGFR- 1 and VEGFR-2) and are activated by VEGF. The third is present on lymphatic endothelium (VEGFR-3), binds VEGF-C, and is involved in lymphangiogenesis. Increased VEGF expression has been described in several tumours and has been associated with increased microvessel density in oesophageal squamous carcinoma, ${ }^{16}$ renal cell carcinoma, ${ }^{17}$ non-small cell lung cancer, ${ }^{18}$ and prostatic adenocarcinoma. ${ }^{19}$ Angiopoietin 1 is one of a family of four related molecules, and it binds the Tie 2 receptor present on endothelial cells, resulting in the stabilisation of vessels. Angiopoietin 2 acts as an antagonist to angiopoietin 1 at this receptor, resulting in the destabilisation of microvessels that is required in the early stages of angiogenesis. Increased angiopoietin 2 expression has been found in non-small cell lung cancer, ${ }^{20}$ uveal melanoma, ${ }^{21}$ and hepatocellular carcinoma, ${ }^{22}$ and this molecule is coexpressed with VEGF. ${ }^{20}{ }^{21}$

In addition to specific angiogenic factors, there are several multifunctional growth factors that have angiogenic activity. Thymidine phosphorylase (also known as platelet derived endothelial cell growth factor) is an intracellular enzyme that regulates thymidine metabolism and has angiogenic activity, stimulating both endothelial cell proliferation and chemotaxis. It can also confer resistance to hypoxia induced apoptosis. It is expressed by both tumour and stromal cells in a variety of tumours, including breast cancer. ${ }^{23} \mathrm{bFGF}$ is produced by tumour, stromal, and endothelial cells, and stimulates endothelial cell proliferation, protease production, chemotaxis, and vascular tube formation. ${ }^{24}$

Extracellular proteases including the matrix metalloproteases (MMPs) and cathepsins are also produced by tumour, stromal, and endothelial cells, resulting in the degradation of the basement membrane and the extracellular matrix. ${ }^{25}$ Not only does this facilitate capillary formation, it also releases angiogenic and anti-angiogenic factors bound to the stroma and exposes integrin binding sites involved in cell migration. For example, b-FGF binds the extracellular matrix, so providing an inactive pool of $\mathrm{b}-\mathrm{FGF}$, which is released and activated by proteolytic enzymes such as MMP- $2 .^{26}$

Finally, elements of the coagulation system, including thrombin, plasmin, and TF have also been shown to encourage new vessel growth. ${ }^{27} \mathrm{TF}$ is expressed by tumour cells ${ }^{28}$ and tumour associated vasculature, ${ }^{29}$ where it can activate the extrinsic coagulation pathway, resulting in thrombin production. Thrombin catalyses the deposition of fibrin, which aids endothelial cell migration and also has a mitogenic effect. ${ }^{30}$
Urokinase-type plasminogen activator, present in the extracellular matrix and on the surface of endothelial cells, converts plasminogen to plasmin, which in turn activates MMPs. ${ }^{31}$

In normal tissues, angiogenic inhibitors exist to prevent exuberant new vessel growth. These inhibitors have been described and studied more recently and include circulating factors, such as angiostatin and endostatin, and the extracellular protein TSP-1. Angiostatin is a cleavage product of plasminogen and is produced by some primary tumours, either directly or via enzymatic cleavage of plasminogen. It has been shown to inhibit endothelial cell proliferation and the growth of metastases, presumably by inhibiting angiogenesis, a phenomenon described as tumour dormancy. ${ }^{32}$ Removal of the primary tumour results in the loss of circulating angiostatin and unfettered growth of the metastases. Endostatin is a protein fragment of collagen XVIII that also inhibits endothelial cell proliferation. ${ }^{33}$

The TSPs are a family of five extracellular proteins that show varied tissue distribution. TSP-1 expression has been reported in squamous cell carcinoma, ${ }^{34}$ melanoma, ${ }^{35}$ and breast carcinoma. ${ }^{85}{ }^{36}$ It is localised to the cell surface and to the extracellular matrix. ${ }^{37}$ There is a close relation between the expression of TSP-1 and wild-type p53, loss of wild-type p53 being associated with loss of TSP-1 expression. ${ }^{38}$ TSP-1 has been shown to have both angiogenic and anti-angiogenic effects. The molecule possesses an integrin recognition site that recognises a specific amino acid sequence (RGD) present within integrin molecules including $\alpha \mathrm{V} \beta 3$. This integrin is expressed by newly formed vessels and facilitates endothelial cell survival ${ }^{39}{ }^{40}$ and adhesion to components of the extracellular matrix, including TSP-1, fibronectin, and fibrin. However, TSP-1 can bind heparin sulfate on the cell surface and in the extracellular matrix, thereby inhibiting the action of heparin dependent growth factors, such as bFGF. ${ }^{41}$ TSP-1 also binds transforming growth factor $\beta$, which itself has both proangiogenic and anti-angiogenic effects. ${ }^{42}$

\section{MEASUREMENT OF TUMOUR ANGIOGENESIS}

The study of angiogenesis in tumour biology is fraught with difficulty, particularly in the assessment of vascularity in histological sections from archival tissue. In this instance one is essentially measuring a dynamic three dimensional process in a static two dimensional preselected section and there has been much debate in the literature as to which counting method most accurately measures tumour microvessel density.

A variety of markers for the identification of microvessels in histological sections are available, the most commonly used being factor VIII, CD31, and CD34. E-9 is reported to be a marker of activated/proliferating endothelial cells, ${ }^{43}$ and may more accurately reflect angiogenic activity within a tumour.

Methods of counting include manual counting of vascular hotspots as described by Weidner et $a l^{44}$ assessment with a Chalkey graticule, and computerised image analysis, ${ }^{45}$ with reported good correlation between these methods. ${ }^{45-47}$ The relative merits of the various counting methods and available antibodies have been well reviewed in the literature, ${ }^{48} 49$ and there are published recommendations for the assessment of tumour microvessel density in histological sections. ${ }^{50}$

A fully automated method of counting microvessel density has been described by Belien et al. ${ }^{51}$ Using image analysis, each vessel in a whole tumour section was mapped to create a microvessel map of the tumour. This was analysed by a program that automatically searched for microvessel "hotspots", as defined by the programmer, and then proceeded to count the vessels in that hot spot. They found that although for the same hotspots the correlation between manual and automated counts was good, the hotspots selected by the automated system had a greater microvessel count than those selected manually. These results suggest that 
full automation could result in greater accuracy, objectivity, and reproducibility of microvessel counts.

Comparison between studies in which counting protocols vary is difficult. Some would argue that the expression of angiogenic factors is a better measure of tumour angiogenesis than tumour microvessel density. However, new vessel growth depends on the net effect of multiple growth factors and inhibitors, and interactions between tumour cells, inflammatory cells, stromal cells, and endothelial cells. Tumour microvessels are the visible manifestation of this activity and quantitation of microvessels remains a valid measure of tumour angiogenesis.

\section{ANGIOGENESIS AND INVASIVE BREAST CARCINOMA}

Notwithstanding these limitations, there is a large body of evidence in the literature that angiogenesis has prognostic relevance in certain tumours, including invasive breast cancer. Weidner and colleagues ${ }^{44}$ demonstrated a correlation between tumour microvessel density in breast cancer and the presence of axillary lymph node and distant metastases. Further studies have confirmed these results and have shown that tumour microvessel density in invasive breast carcinoma is an independent prognostic factor for disease free and overall survival. ${ }^{52-55}$ Some studies have failed to show an association between microvessel density and prognosis, possibly because of deviation from recommended counting methods. ${ }^{56}$

The angiogenic factors studied in invasive breast cancer include VEGF and thymidine phosphorylase; both in relation to microvessel density and as independent prognostic indicators. Increased expression of VEGF by breast cancer cells has been correlated with increased microvessel density ${ }^{57}$ and poorer prognosis in node positive ${ }^{59}$ and node negative patients. ${ }^{60}$ The expression and activation of one of the VEGF receptors, VEGFR-2, is increased in invasive breast cancer. ${ }^{15} 61$ One study showed the association between VEGF and microvessel density to hold only for invasive ductal carcinoma and not for invasive lobular carcinoma, ${ }^{58}$ and others have not found an association between VEGF expression and vascularity. ${ }^{14}$ Thymidine phosphorylase is also upregulated in invasive breast carcinoma and is expressed by both tumour cells and inflammatory cells in the surrounding stroma. Expression of thymidine phosphorylase is associated with increased microvessel density and has been reported to have prognostic value. ${ }^{62}$ Other angiogenic factors that are increased in invasive ductal carcinoma include bFGF, ${ }^{63} \mathrm{MMPs}^{64}$ and the integrin $\alpha \mathrm{V} \beta 3$, which shows increased expression on tumour vessels. ${ }^{65}$

There has been less work on anti-angiogenic factors in breast carcinomas. Invasive ductal and lobular carcinomas show different patterns of TSP-1 expression. TSP-1 protein has been consistently localised to the desmoplastic stroma around invasive ductal carcinoma, with relatively little staining of tumour cells. ${ }^{366-70}$ The converse is seen in invasive lobular carcinoma. ${ }^{36}$ Although TSP-1 is reported to have an antiangiogenic effect in breast cancer cell lines, ${ }^{8}$ studies of TSP-1 expression in tissue sections from invasive breast cancer show both positive ${ }^{66} 70$ and negative $e^{35}{ }^{71}$ associations between TSP-1 and microvessel density. ${ }^{72}$ Interestingly, one study by Pazouki et al found that human breast cancers fell into two groups when TSP-1 and microvessel density were assessed, one group showing a positive association between TSP-1 and microvessel density and the other a negative association. ${ }^{73}$

\section{ANGIOGENESIS AND IN SITU CARCINOMA}

It is becoming increasingly apparent that the angiogenic phenotype may be acquired early in tumour evolution and increased angiogenic activity has been reported in preinvasive neoplastic lesions of the cervix, ${ }^{74-76}$ vulva, ${ }^{77}$ bladder, ${ }^{78}$ oral mucosa, ${ }^{79}$ and lung. ${ }^{80} 81$ Angiogenesis in in situ tumours may differ from that seen in invasive tumours. The increase in vessels is seen in the normal stroma around or beneath the in situ tumour, rather than between clusters of tumour cells, as in invasive disease. Therefore, in in situ tumours, angiogenic factors produced by inflammatory and stromal cells may play a greater role than those derived from the tumour cells themselves. Two patterns of vascularity have been described in several in situ tumours. Studies of cervical intraepithelial neoplasia (CIN) have demonstrated an increase in vessels lying immediately adjacent to the basement membrane, at the interface between the abnormal squamous epithelium and the underlying stroma, and an increase in vessels within this underlying stroma. ${ }^{75}$ Similar patterns have been described in ductal carcinoma in situ (DCIS), as discussed below. In parallel with invasive disease, increased microvessel density is associated with the transition from low grade to high grade in situ lesions, suggesting that angiogenesis may play a role in tumour progression at this stage. For example, in the cervix, both vascular patterns were significantly increased in high grade CIN as compared with low grade lesions and benign squamous epithelium. ${ }^{74}$ This increase was also correlated with increased expression of VEGF mRNA by the neoplastic cells. ${ }^{75}$ In the lung, dysplastic bronchial mucosa and carcinoma in situ had significantly higher microvessel counts than did normal, hyperplastic, or metaplastic epithelium. ${ }^{80} 81$

\section{ANGIOGENESIS IN DCIS \\ Morphological patterns and relation to histological characteristics}

In a series of breast carcinomas, Weidner and colleagues ${ }^{44}$ described a ring of small vessels around ducts involved by DCIS. Two patterns of vascularity around DCIS were subsequently described by Guidi et al, resembling those seen in CIN. The first of these comprised a cuff or necklace of microvessels immediately adjacent to the basement membrane of ducts with DCIS and the second a diffuse increase in stromal microvessels surrounding involved duct spaces. ${ }^{82}$ The presence of microvessels was assessed both semiquantitatively and quantitatively with good correlation. Increased stromal microvessel density was associated with high histological grade $^{83}$ and with the presence of comedo necrosis. ${ }^{82} 84$ Engels and colleagues ${ }^{83}$ also reported an association between the presence of a cuff of vessels and the grade of DCIS, but this has not been confirmed by other studies. ${ }^{82} 86$

\section{Angiogenic factors}

Increased VEGF mRNA expression has been demonstrated in tumour cells of DCIS compared with neighbouring benign epithelium, and was associated with an increase in stromal microvessel density around DCIS ducts. ${ }^{87}$ The same group reported increased VEGF mRNA expression in high grade DCIS and in DCIS with a cuff of microvessels, although these associations were not significant. Increased expression of VEGF receptors has also been found on endothelial cells around DCIS ${ }^{88}$ Other groups have failed to find an association between VEGF expression by tumour cells in DCIS and stromal microvessel density. ${ }^{58}{ }^{84}$ However, hypoxia inducible factor $1 \alpha$ is increased in DCIS and associated with increased stromal microvessel density. ${ }^{89}$ Under hypoxic conditions hypoxia inducible factor $1 \alpha$ is stabilised in the nucleus and transactivates various genes, including the VEGF gene.

In DCIS, thymidine phosphorylase is expressed by both tumour and stromal cells, in contrast to VEGF, which is primarily of tumour origin. Studies to date have produced varied results. Engels et al reported the expression of thymidine phosphorylase by DCIS tumour cells, which showed a significant association with the presence of a cuff of vessels, but was not associated with stromal microvessel density. ${ }^{90}$ Other studies have failed to confirm the association between thymidine phosphorylase expression in DCIS tumour 
cells and a cuff of microvessels. ${ }^{84}{ }^{91}$ Lee and colleagues ${ }^{91}$ investigated the expression of thymidine phosphorylase by inflammatory cells in the stroma around DCIS. They found an association between the expression of thymidine phosphorylase by inflammatory cells and stromal microvessel density, complimenting their previous findings of an association between perivascular inflammation and increased stromal vascularity in DCIS. ${ }^{86}$ These data highlight the potential importance of inflammatory cells as a source of angiogenic factors in DCIS.

TF is expressed by DCIS tumour cells and on vascular endothelial cells in the surrounding stroma. ${ }^{92}$ This may be secondary to VEGF, which is also expressed by endothelial cells around DCIS, and which is known to induce endothelial cell expression of $\mathrm{TF}^{93}$ Thus, the coagulation system, elements of which are implicated in new vessel growth (for example, thrombin and fibrin), probably plays a role in angiogenesis around DCIS. In addition, MMP mRNAs are expressed in in situ and in invasive breast carcinomas. The MMP mRNAs expressed include MMP-2, MMP-7, MMP-9, and MMP-11, and expression has been described variably in tumour cells and stromal fibroblasts..$^{69495}$

\section{Anti-angiogenic factors}

The expression of both TSP-1 protein and mRNA has been described in several studies of between two and 18 cases of DCIS. ${ }^{36}{ }^{66} 70$ TSP-1 protein was localised to the basement membrane around involved duct spaces and the immediately adjacent collagen. In one study, the TSP-1 mRNA signal was localised to the myoepithelial cells bordering DCIS ducts, suggesting that these cells are the source of TSP- $1 .{ }^{68}$

In a larger series of 58 patients with DCIS, we confirmed the pattern of TSP-1 protein expression as described, and also saw a pattern of stromal staining between involved ducts similar to that seen in invasive carcinoma. ${ }^{96}$ Our results are supported by the study of Brown et al, in which TSP-1 mRNA was identified in stromal cells in the vicinity of ducts involved by DCIS ${ }^{67}$ We failed to find an association between TSP expression (both stromal and periductal) and either the presence of a cuff of vessels or stromal microvessel density. These data suggest that other factors have a more influential role in angiogenesis around DCIS.

As in studies of invasive carcinomas, studies of angiogenic factors in DCIS have produced conflicting results. This may be the result of differing sample size, lack of correlation between mRNA and protein expression, or variation in vessel counting methods. Study results may also be influenced by difficulties in distinguishing new or remodelled vessels from pre-existing vessels and the evaluation of angiogenic factors in isolation, which does not take account of synergistic/antagonistic interactions between these factors.

\section{CONCLUSION}

The angiogenic phenotype of breast carcinoma, as in other tumours, is acquired early in tumorigenesis while the tumour is still in situ. It is likely that multiple angiogenic factors are involved and the two vascular patterns in DCIS may arise by different mechanisms. For example, the cuff of vessels may arise in response to an angiogenic factor produced by tumour cells that diffuses only a short distance or is inactivated by factors in the surrounding stroma. The stromal vessels may be induced by angiogenic factors produced by tumour and stromal cells (for example, tumour associated macrophages), in response to some as yet unknown factor produced by the tumour. Invasive tumours arising from highly vascular in situ lesions are likely to be highly angiogenic, although this will depend not only on tumour phenotype, but also on the influence of the surrounding stroma. It may be that the full angiogenic potential of a tumour is not realised until it becomes invasive, thereby permitting interaction with stromal cells and the extracellular matrix.

Considering the invasive potential of high grade DCIS, the reported association between increased microvessel density, high histological grade, and comedo necrosis suggests that angiogenesis may influence the progression of DCIS to invasive carcinoma. The acquisition of invasive properties by in situ tumour is a complex process, involving interactions between tumour cells, endothelial cells, stroma, and the extracellular matrix. The expression of MMPs by both tumour and endothelial cells is upregulated by several growth factors including VEGF, epidermal growth factor, and platelet derived growth factor. Paracrine interactions between endothelial and tumour cells may play an important role in tumour progression-for example, in the transition of radial growth phase melanoma to thick vertical growth phase. ${ }^{6}$ Skobe et al showed that the invasiveness of a human squamous carcinoma cell line transplanted into mice was associated with ongoing angiogenesis, and that halting angiogenesis (using antibodies to VEGFR-2 expressed on endothelial cells) resulted in reversion of the tumour cells to a non-invasive phenotype. ${ }^{97}$ They also found that overexpression of VEGF by these tumour cells resulted in an accelerated rate of tumour growth, with the development of an invasive border. In contrast, cell lines that did not overexpress VEGF showed slower growth, with a pushing tumour border. ${ }^{98}$ Similarly, Brooks et al found that in human breast cancer implants, inhibition of angiogenesis using antibodies to the integrin $\alpha \mathrm{V} \beta 3$ (which like VEGFR-2 is expressed on endothelial cells) resulted in reduced tumour invasiveness. ${ }^{99}$

Our knowledge of DCIS and approach to histological assessment have advanced considerably in the past two decades, and it is known that patients with large, high grade lesions are at greater risk of developing invasive carcinoma. It is not possible to predict which lesions will progress, and all patients with DCIS require surgery and many undergo radiotherapy. The possibility that angiogenesis may be important in the transition to invasive disease is exciting. Currently, the available information is incomplete and there is a need for detailed profiling of angiogenic factors expressed in both DCIS and invasive carcinoma, possibly with computer modelling of the multiple possible pathways and paracrine interactions that govern angiogenesis. Knowledge of the angiogenic profile of an individual tumour may improve our ability to prognosticate and the development of specific anti-angiogenic therapeutic regimens may provide an alternative to current treatment options both for in situ and invasive disease.

\section{Authors' affiliations}

A Rice, Royal Brompton Hospital, Sydney Street, London SW3 6NP, UK C M Quinn, Irish National Breast Screening Programme and St Vincent's University Hospital, Elm Park, Donnybrook, Dublin 4, Ireland

\section{REFERENCES}

1 Folkman J. Angiogenesis research: from laboratory to clinic. Forum (Genova) 1999;9:59-62.

2 Kerbel RS. Tumor angiogenesis: past, present and the near future. Carcinogenesis 2000;21:505-15.

3 Carmeliet P. Mechanisms of angiogenesis and arteriogenesis. Nat Med 2000;6:389-95

4 Patan S, Munn LL, Jain RK. Intussusceptive microvascular growth in a human colon adenocarcinoma xenograft: a novel mechanism of tumor angiogenesis. Microvasc Res 1996;51:260-72.

5 Rafii S. Circulating endothelial precursors: mystery, reality, and promise. J Clin Invest 2000;105:17-19

6 Rak J, Filmus J, Kerbel RS. Reciprocal paracrine interactions between tumour cells and endothelial cells: the "angiogenesis progression" hypothesis. Eur J Cancer 1996:32A:2438-50.

7 Rak J, Klement G. Impact of oncogenes and tumor suppressor genes on deregulation of hemostasis and angiogenesis in cancer. Cancer Metastasis Rev 2000;19:93-6.

8 Volpert OV, Stellmach V, Bouck N. The modulation of thrombospondin and other naturally occurring inhibitors of angiogenesis during tumor progression. Breast Cancer Res Treat 1995;36:119-26. 


\section{Take home messages}

- Angiogenesis, the formation of new vessels from existing vasculature, is essential for tumour growth and metastasis

- Increased angiogenic activity has been documented in a variety of pre-invasive neoplastic conditions, suggesting that the angiogenic phenotype may be acquired early in tumour evolution

- The angiogenic process relies on a complex interaction between tumour, stromal, endothelial and inflammatory cells, growth factors, and the extracellular matrix, which is regulated by pro-angiogenic and anti-angiogenic factors

- Tumour microvessels are the visible manifestation of this complex interaction and quantitation of microvessels in tissue sections is a valid measure of tumour angiogenesis

- Microvessel density is an independent prognostic indicator in invasive breast cancer

- A variety of angiogenic factors are produced by ductal carcinoma in situ, which are variously associated with changes in vascularity, and may facilitate the transition to invasive carcinoma

- The development of specific anti-angiogenic regimens may provide an alternative to current treatment protocols for breast cancer

9 Iliopoulos $\mathrm{O}$, Levy AP, Jiang $\mathrm{C}$, et al. Negative regulation of hypoxia-inducible genes by the von Hippel-Lindau protein. Proc Nat Acad Sci U S A 1996:93:10595-9.

10 de Jong JS, van Diest PJ, Baak JP. Hot spot microvessel density and the mitotic activity index are strong additional prognostic indicators in invasive breast cancer. Histopathology 2000;36:306-12.

11 Yamane A, Seetharam L, Yamaguchi S, et al. A new communication system between hepatocytes and sinusoidal endothelial cells in liver through vascular endothelial growth factor and Flt tyrosine kinase receptor family (Flt-1 and KDR/Flk-1). Oncogene 1994;9:2683-90.

12 Sunderkotter C, Steinbrink K, Goebeler M, et al. Macrophages and angiogenesis. J Leukoc Biol 1994;55:410-22.

13 Lwaleed BA, Bass PS, Cooper AJ. The biology and tumour-related properties of monocyte tissue factor. J Pathol 2001;193:3-12.

14 de Jong JS, van Diest PJ, van der Valk P, et al. Expression of growth factors, growth-inhibiting factors, and their receptors in invasive breas cancer. II: Correlations with proliferation and angiogenesis. J Pathol 1998;184:53-7.

15 de Jong JS, van Diest PJ, van der Valk P, et al. Expression of growth factors, growth inhibiting factors, and their receptors in invasive breas cancer. I: An inventory in search of autocrine and paracrine loops. J Pathol 1998;184:44-52.

16 Koide N, Nishio A, Hiraguri M, et al. Coexpression of vascular endothelial growth factor and p53 protein in squamous cell carcinoma of the esophagus. Am J Gastroenterol 2001;96:1733-40.

17 Lee JS, Kim HS, Jung JJ, et al. Expression of vascular endothelial growth factor in renal cell carcinoma and the relation to angiogenesis and p53 protein expression. J Surg Oncol 2001;77:55-60.

18 Han H, Silverman JF, Santucci TS, et al. Vascular endothelial growth factor expression in stage I non-small cell lung cancer correlates with neoangiogenesis and a poor prognosis. Ann Surg Oncol 2001;8:72-9.

19 Strohmeyer D, Rossing C, Baverfeind A, et al. Vascular endothelia growth factor and its correlation with angiogenesis and p53 expression in prostate cancer. Prostate 2000;45:216-24.

20 Wong MP, Chan SY, Fu KH, et al. The angiopoietins, tie 2 and vascular endothelial growth factor are differentially expressed in the transformation of normal lung to non-small cell lung carcinomas. Lung Cancer 2000;29:11-22.

21 liland SA, Jager M, Heijdra BM, et al. Expression of angiogenic and immunosuppressive factors by uveal melanoma cell lines. Melanoma Res 1999;9:445-50

22 Tanaka S, Mori M, Sakamoto $Y$, et al. Biologic significance of angiopoietin-2 expression in human hepatocellular carcinoma. J Clin Invest 1999; 103:341-5.

23 Fox SB, Westwood M, Moghaddam A, et al. The angiogenic factor platelet-derived endothelial cell growth factor/thymidine phosphorylase is up-regulated in breast cancer epithelium and endothelium. Br J Cancer 1996:73:275-80.

24 Cross MJ, Claesson-Welsh L. FGF and VEGF function in angiogenesis: signalling pathways, biological responses and therapeutic inhibition. Trends Pharmacol Sci 2001;22:201-7.

25 Stetler-Stevenson WG, Hewitt R, Corcoran M. Matrix metalloproteinases and tumor invasion: from correlation and causality to the clinic. Semin Cancer Biol 1996;7:147-54

26 Vlodavsky I, Bar-Shavit R, Ishai-Michaeli R, et al. Extracellular sequestration and release of fibroblast growth factor: a regulatory mechanism? Trends Biochem Sci 1991;16:268-71.

27 Francis JL, Biggerstaff J, Amirkhosravi A. Hemostasis and malignancy. Semin Thromb Hemost 1998;24:93-109.
28 Callander NS Varki N, Rao LV. Immunohistochemical identification of tissue factor in solid tumors. Cancer 1992;70:1194-201.

29 Contrino J, Hair G, Kreutzer DL, et al. In situ detection of tissue factor in vascular endothelial cells: correlation with the malignant phenotype of human breast disease. Nat Med 1996;2:209-15.

30 Kadish JL, Butterfield CE, Folkman J. The effect of fibrin on cultured vascular endothelial cells. Tissue Cell 1979;11:99-108.

31 Matrisian LM. Cancer biology: extracellular proteinases in malignancy. Curr Biol 1999; 9:R776-8

32 O'Reilly MS, Holmgren L, Shing Y, et al. Angiostatin: a circulating endothelial cell inhibitor that suppresses angiogenesis and tumor growth. Cold Spring Harb Symp Quant Biol 1994;59:471-82.

33 O'Reilly MS, Boehm T, Shing Y, et al. Endostatin: an endogenous inhibitor of angiogenesis and tumor growth. Cell 1997;88:277-85

34 Riser BL, Varani J, O'Rourke K, et al. Thrombospondin binding by human squamous carcinoma and melanoma cells: relationship to biological activity. Exp Cell Res 1988;174:319-29.

35 Zabrenetzky V, Harris CC, Steeg PS, et al. Expression of the extracellular matrix molecule thrombospondin inversely correlates with malignant progression in melanoma, lung and breast carcinoma cell lines. Int J Cancer 1994;59:191-5.

36 Wong SY, Purdie AT, Han P. Thrombospondin and other possible related matrix proteins in malignant and benign breast disease. An immunohistochemical study. Am J Pathol 1992;140:1473-82.

37 McKeown-Longo PJ, Hanning R, Mosher DF. Binding and degradation of platelet thrombospondin by cultured fibroblasts. J Cell Biol 1984;98:22-8.

38 Dameron KM, Volpert OV, Tainsky MA, et al. Control of angiogenesis in fibroblasts by $\mathrm{p} 53$ regulation of thrombospondin-1. Science 1994;265: 1582-4.

39 Brooks PC, Clark RA, Cheresh DA. Requirement of vascular integrin alpha $v$ beta 3 for angiogenesis. Science 1994;264:569-71.

40 Brooks PC, Montgomery AM, Rosenfeld M, et al. Integrin alpha v beta 3 antagonists promote tumor regression by inducing apoptosis of angiogenic blood vessels. Cell 1994;79:1157-64

41 Taraboletti G, Roberts D, Liotta LA, et al. Platelet thrombospondin modulates endothelial cell adhesion, motility, and growth: a potential angiogenesis regulatory factor. J Cell Biol 1990;111:765-72.

42 Murphy-Ullrich JE, Schultz-Cherry S, Hook M. Transforming growth factor-beta complexes with thrombospondin. Mol Biol Cell 1992;3:181-8

43 Wang JM, Kumar S, Pye D, et al. A monoclonal antibody detects heterogeneity in vascular endothelium of tumours and normal tissues. Int J Cancer 1993:54:363-70.

44 Weidner N, Semple JP, Welch WR, et al. Tumor angiogenesis and metastasis - correlation in invasive breast carcinoma. N Engl J Med $1991 ; 324: 1-8$

45 Fox SB, Leek RD, Weekes MP, et al. Quantitation and prognostic value of breast cancer angiogenesis: comparison of microvessel density, Chalkley count, and computer image analysis. J Pathol 1995; 177:275-83.

46 Pendleton N, Pazouki S, Heerkens E, et al. Relationships between different measurements of vascularity and clinico-pathological parameters in breast cancer. Anticancer Res 1998;18:4565-8.

47 Hansen S, Grabau DA, Rose C, et al. Angiogenesis in breast cancer: a comparative study of the observer variability of methods for determining microvessel density. Lab Invest 1998;78:1563-73.

48 Barbareschi M, Gasparini G, Morelli L, et al. Novel methods for the determination of the angiogenic activity of human tumors. Breast Cancer Res Treat 1995:36:181-92

49 Weidner N. Current pathologic methods for measuring intratumoral microvessel density within breast carcinoma and other solid tumors. Breast Cancer Res Treat 1995;36:169-80.

50 Vermeulen PB, Gasparini G, Fox SB, et al. Quantification of angiogenesis in solid human tumours: an international consensus on the methodology and criteria of evaluation. Eur J Cancer 1996;32A:247484

51 Belien JA, Somi S, de Jong JS, et al. Fully automated microvessel counting and hot spot selection by image processing of whole tumour sections in invasive breast cancer. J Clin Pathol 1999:52:184-92.

52 Horak ER, Leek R, Klenk N, et al. Angiogenesis, assessed by platelet/endothelial cell adhesion molecule antibodies, as indicator of node metastases and survival in breast cancer. Lancet 1992;340: $1120-4$

53 Bosari S, Lee AK, DeLellis RA, et al. Microvessel quantitation and prognosis in invasive breast carcinoma. Hum Pathol 1992:23:755-61.

54 Fox SB, Leek RD, Smith K, et al. Tumor angiogenesis in node-negative breast carcinomas - relationship with epidermal growth factor receptor, estrogen receptor, and survival. Breast Cancer Res Treat 1994;29:109-16.

55 Weidner N, Folkman J, Pozza F, et al. Tumor angiogenesis: a new significant and independent prognostic indicator in early-stage breast carcinoma. J Natl Cancer Inst 1992;84:1875-87.

56 Fox SB. Tumour angiogenesis and prognosis. Histopathology 1997;30:294-301.

57 Toi $M$, Inada $K$, Hoshina S, et al. Vascular endothelial growth factor and platelet-derived endothelial cell growth factor are frequently coexpressed in highly vascularized human breast cancer. Clin Cancer Res 1995; 1:961-4.

58 Lee AH, Dublin EA, Bobrow LG, et al. Invasive lobular and invasive ductal carcinoma of the breast show distinct patterns of vascular endothelial growth factor expression and angiogenesis. J Pathol 1998; 185:394-401. 
59 Gasparini G, Toi M, Miceli $R$, et al. Clinical relevance of vascular endothelial growth factor and thymidine phosphorylase in patients with node-positive breast cancer treated with either adjuvant chemotherapy or hormone therapy. Cancer J Sci Am 1999:5:101-1 1.

60 Gasparini G, Toi M, Gion M, et al. Prognostic significance of vascular endothelial growth factor protein in node-negative breast carcinoma. $J$ Natl Cancer Inst 1997:89:139-47.

61 Kranz A, Matffeldt T, Waltenberger J. Molecular mediators of tumor angiogenesis: enhanced expression and activation of vascular endothelial growth factor receptor KDR in primary breast cancer. Int $J$ Cancer 1999:84:293-8.

62 Toi $M$, Ueno T, Matsumoto $H$, et al. Significance of thymidine phosphorylase as a marker of protumor monocytes in breast cancer. Clin Cancer Res 1999:5:1131-7.

63 Visscher DW, DeMattia F, Ottosen S, et al. Biologic and clinical significance of basic fibroblast growth factor immunostaining in breast carcinoma. Mod Pathol 1995;8:665-70.

64 Heppner KJ, Matrisian LM, Jensen RA, et al. Expression of most matrix metalloproteinase family members in breast cancer represents a tumor-induced host response. Am J Pathol 1996;149:273-82.

65 Gasparini G, Brooks PC, Biganzoli E, et al. Vascular integrin alpha(v)beta3: a new prognostic indicator in breast cancer. Clin Cancer Res 1998;4:2625-34.

66 Bertin N, Clezardin P, Kubiak R, et al. Thrombospondin-1 and -2 messenger RNA expression in normal, benign, and neoplastic human breast tissues: correlation with prognostic factors, tumor angiogenesis, and fibroblastic desmoplasia. Cancer Res 1997;57:396-9.

67 Brown LF, Guidi AJ, Schnitt SJ, et al. Vascular stroma formation in carcinoma in situ, invasive carcinoma, and metastatic carcinoma of the breast. Clin Cancer Res 1999;5:1041-56.

68 Clezardin P, Frappart L, Clerget M, et al. Expression of thrombospondin (TSP1) and its receptors (CD36 and CD51) in normal, hyperplastic, and neoplastic human breast. Cancer Res 1993;53:1421-30.

69 Serre CM, Clezardin P, Frappart L, et al. Distribution of thrombospondin and integrin alpha $\mathrm{V}$ in DCIS, invasive ductal and lobular human breast carcinomas. Analysis by electron microscopy. Virchows Arch 1995;427:365-72

70 Tuszynski GP, Nicosia RF. Localization of thrombospondin and its cysteine-serine-valine-threonine-cysteine-glycine-specific receptor in human breast carcinoma. Lab Invest 1994;70:228-33.

71 Weinstat-Saslow DL, Zabrenetzky VS, VanHoutte K, et al. Transfection of thrombospondin 1 complementary DNA into a human breast carcinoma cell line reduces primary tumor growth, metastatic potential, and angiogenesis. Cancer Res 1994;54:6504-11.

72 Steward MA, Rice AJ, Roberts D, et al. Thrombospondin-1 in invasive breast cancer and its association with p53 expression, microvessel density and clinical outcome. J Pathol 1998;184(suppl):5A.

73 Pazouki S, Pendleton N, Heerkens E, et al. Biphasic effect of thrombospondin-1 (TSP-1) in the regulation of angiogenesis in human breast carcinoma. Biochem Soc Trans 1996;24:368S

74 Smith-McCune KK, Weidner N. Demonstration and characterization of the angiogenic properties of cervical dysplasia. Cancer Res 1994;54:800-4

75 Guidi AJ, Abu-Jawdeh G, Berse B, et al. Vascular permeability facto (vascular endothelial growth factor) expression and angiogenesis in cervical neoplasia. J Natl Cancer Inst 1995;87:1237-45.

76 Obermair A, Bancher-Todesca D, Bilgi S, et al. Correlation of vascular endothelial growth factor expression and microvessel density in cervical intraepithelial neoplasia. J Natl Cancer Inst 1997;89:1212-17.

77 Bancher-Todesca D, Obermair A, Bilgi S, et al. Angiogenesis in vulvar intraepithelial neoplasia. Gynecol Oncol 1997;64:496-500.

78 Chodak GW, Haudenschild C, Gittes RF, et al. Angiogenic activity as a marker of neoplastic and preneoplastic lesions of the human bladder. Ann Surg 1980;192:762-71.
79 Macluskey M, Chandrachud LM, Pazouki S, et al. Apoptosis, proliferation, and angiogenesis in oral tissues. Possible relevance to tumour progression. J Pathol 2000;191:368-75

80 Fontanini G, Vignati S, Bigini D, et al. Neoangiogenesis: a putative marker of malignancy in non-small-cell lung cancer (NSCLC) development. Int J Cancer 1996:67:615-19.

81 Fisseler-Eckhoff A, Rothstein D, Muller KM. Neovascularization in hyperplastic, metaplastic and potentially preneoplastic lesions of the bronchial mucosa. Virchows Arch 1996;429:95-100.

82 Guidi AJ, Fischer L, Harris JR, et al. Microvessel density and distribution in ductal carcinoma in situ of the breast. J Natl Cancer Inst 1994;86:614-19.

83 Engels K, Fox SB, Whitehouse RM, et al. Distinct angiogenic patterns are associated with high-grade in situ ductal carcinomas of the breast. $J$ Pathol 1997; 181:207-12.

84 Heffelfinger SC, Miller MA, Yassin R, et al. Angiogenic growth factors in preinvasive breast disease. Clin Cancer Res 1999;5:2867-76.

85 Zolota V, Gerokosta A, Melachrinou M, et al. Microvessel density, proliferating activity, p53 and bcl-2 expression in in situ ductal carcinoma of the breast. Anticancer Res 1999;19:3269-74.

86 Lee AH, Happerfield LC, Bobrow LG, et al. Angiogenesis and inflammation in ductal carcinoma in situ of the breast. J Pathol 1997; 181:200-6.

87 Guidi AJ, Schnitt SJ, Fischer L, et al. Vascular permeability factor (vascular endothelial growth factor) expression and angiogenesis in patients with ductal carcinoma in situ of the breast. Cancer 1997:80: 1945-53.

88 Brown LF, Berse B, Jackman RW, et al. Expression of vascular permeability factor (vascular endothelial growth factor) and its receptors in breast cancer. Hum Pathol 1995;26:86-91.

89 Bos R, Zhong H, Hanrahan CF, et al. Levels of hypoxia-inducible factor-1 alpha during breast carcinogenesis. J Natl Cancer Inst 2001:93:309-14.

90 Engels K, Fox SB, Whitehouse RM, et al. Up-regulation of thymidine phosphorylase expression is associated with a discrete pattern of angiogenesis in ductal carcinomas in situ of the breast. J Pathol 1997; 182:414-20.

91 Lee AH, Dublin EA, Bobrow LG. Angiogenesis and expression of thymidine phosphorylase by inflammatory and carcinoma cells in ductal carcinoma in situ of the breast. J Pathol 1999;187:285-90.

92 Vrana JA, Stang MT, Grande JP, et al. Expression of tissue factor in tumor stroma correlates with progression to invasive human breast cancer: paracrine regulation by carcinoma cell-derived members of the transforming growth factor beta family. Cancer Res 1996:56:5063-70.

93 Clauss M, Gerlach M, Gerlach H, et al. Vascular permeability factor: a tumor-derived polypeptide that induces endothelial cell and monocyte procoagulant activity, and promotes monocyte migration. J Exp Med 1990; 172:1535-45

94 Brummer O, Athar S, Riethdorf L, et al. Matrix-metalloproteinases 1, 2 , and 3 and their tissue inhibitors 1 and 2 in benign and malignant breast lesions: an in situ hybridization study. Virchows Arch 1999:435:56673.

95 Lee KS, Rha SY, Kim SJ, et al. Sequential activation and production of matrix metalloproteinase-2 during breast cancer progression. Clin Exp Metastasis 1996:14:512-19.

96 Rice AJ, Steward MA, Horgan K, et al. Thrombospondin-1 expression and angiogenesis in ductal carcinoma in-situ of the breast. J Pathol 1999; 187 (suppl):26A

97 Skobe M, Rockwell P, Goldstein N, et al. Halting angiogenesis suppresses carcinoma cell invasion. Nat Med 1997;3:1222-7.

98 Detmar M, Velasco P, Richard L, et al. Expression of vascular endothelial growth factor induces an invasive phenotype in human squamous cell carcinomas. Am J Pathol 2000;156:159-67.

99 Brooks PC, Stromblad S, Klemke R, et al. Antiintegrin alpha $v$ beta 3 blocks human breast cancer growth and angiogenesis in human skin. $J$ Clin Invest 1995;96:1815-22. 